\title{
Future Disease-Centered Principles of Endoscopic Treatment
}

\author{
Enqiang Linghu
}

1. Follow the principle of cavity integrity: Place the entry incision, pathway, and target position of the operation in the same cavity as much as possible.

2. Sterile conditions are preferred over unsterile ones.

3. Between conditions with and without chemical stimulation, the latter shall prevail.

4. Natural orifices are preferred over non-natural ones, and the choice of natural orifices should first meet the two principles of (2) and (3).

5. Distance between the entry incision and the target position should be as near as possible, if above principles are met.

6. Endoscopic bleeding-preventing and hemostatic techniques shall be mastered along with alternative measures to ensure almost $100 \%$ of successful hemostasis.

7. Skilled techniques for preventing and closing perforation shall be mastered to restore the integrity and closed state of original cavity.

8. Follow the principle of complete tumor resection and prevention of metastasis.

For the target organ, when there are multiple endoscopic approaches, the approach which meets most of the abovementioned principles would be the preferred option.

E. Linghu $(\bowtie)$

Department of Gastroenterology and Hepatology,

First Medical Center of Chinese PLA General Hospital, Beijing, China 